\title{
Evolución de las características socioeconómicas de los pescadores de Ciénaga, Magdalena
}

\author{
Evolution of the socioeconomic characteristics of fishermen from Ciénaga, Magdalena
}

D0I: http://dx.doi.org/10.21676/23897848.1674

Luis Gabriel Durán-Fernández Economista, Universidad del Magdalena, Santa Marta, Colombia. lgdf07@gmail.com

May Andro Giraldo-Ramírez Economista, Universidad del Magdalena, Santa Marta, Colombia. andro.gr@gmail.com

Álvaro José Núñez- Viana Economista, Universidad del Magdalena, Santa Marta, Colombia. nunesito111@gmail.com

Tipología:

Artículo de Investigación Científica y Tecnológica

Fecha de Recibido: Diciembre 14 de 2015 Fecha de Aceptación: Febrero 17 de 2016

Para citar este artículo: Durán, F. L., Giraldo, R. M., \& Núñez, V. A. (2016). Evolución de las características socioeconómicas de los pescadores de Ciénaga, Magdalena. Clío América, 10 (19), 55 - 64
Resumen: Esta investigación analiza la evolución socioeconómica de los pescadores artesanales del municipio de Ciénaga (Magdalena). La información fue recopilada mediante la realización de dos encuestas, una en el año 2011 (397) perteneciente a un proyecto interno de la Universidad del Magdalena y la otra en el periodo 2014 (199), que permitieron construir una base de datos con información social y económica de esta población objeto de estudio. Los resultados analizados demostraron que no hay grandes avances en la situación socioeconómica de los pescadores artesanales y su núcleo familiar, en el cual los diferentes módulos de las encuestas demostraron estancamientos y desmejora en los diversos niveles de educación alcanzados por los hijos y cónyuges de los encuestados, además un deterioro en la forma de eliminación de desechos porque existe un aumento en la cantidades de hogares que afirmaron que la queman 0 la vierten en el río y la ciénaga.

Palabras Clave: Educación, grupo vulnerable, pesca costera, pobreza, servicio público.

JEL: P36, R11

Abstract: This research analyzes the evolution of artisanal fishermen Economically the municipality of Ciénaga (Magdalena). The information was gathered by conducting two surveys, one in the year 2011 (397) Pertaining to an internal project from the Univerisdad del Magdalena and the other in the period 2014 (199), which allowed build a database with social and Economic information study population. The analyzed results showed no hay great strides in socio-economic status of artisanal fishermen and in which the different modules of household surveys showed the stagnation and deterioration in the various levels of education attained by children and spouses of respondents also deterioration in the way because waste disposal increased numbers of households who reported the burning of or discharged into the river and the swamp.

Keywords: Coastal fisheries, education, poverty, public service, vulnerable group. 


\section{Introducción}

Ciénaga es un municipio localizado a orillas del mar Caribe, en el extremo nororiental de la Ciénaga Grande de Santa Marta; perteneciente al departamento del Magdalena, Colombia. Éste, posee una extensión geográfica de gran magnitud en comparación con los municipios aledaños, con un área de $1.142 .68 \mathrm{~km}^{2}$, se convierte en el segundo territorio de mayor tamaño en el departamento del Magdalena. Con 100.908 habitantes (DANE 2005), es el segundo municipio más poblado del departamento, después de Santa Marta; de cuya población se encontró que el $87 \%$ residía en la cabecera, además el $50,5 \%$ de la misma es de género femenino.

El trabajo determina las principales variables socioeconómicas estudiadas de los pescadores artesanales del municipio de Ciénaga, logrando así observar la evolución que ha tenido la calidad de vida de la población pesquera y de su núcleo familiar, con el fin de determinar si éstas han mejorado o por el contrario se encuentran inmersos en la trampa de la pobreza.

La problemática que tienen que contrarrestar los pescadores artesanales en el desarrollo cotidiano, es la compleja marginalización de sus necesidades fundamentales, asociada a unas privaciones de calidad de vida, donde, además, la mayor fuente del sustento de las familias se deriva de ésta actividad pesquera (Universidad de la Republica, 2011). Sin embargo, ésta investigación contribuirá para la toma de acciones inmediatas, donde a su vez, los actores locales, las instituciones del estado, las ONG, el sector empresarial, la comunidad y las instituciones de investigación y formación como la Universidad de Magdalena, conocerán el estado crítico en que se encuentran los pescadores artesanales de Ciénaga y tendrán las bases para el fomento de políticas de apoyo para el fortalecimiento de esta actividad.

El documento se encuentra fraccionado de la siguiente manera, en la primera parte del trabajo se planteó la metodología utilizada en el artículo. La segunda parte, se realizan unos análisis gráficos sacados de la base de datos de las encuestas realizadas en los años 2011 y 2014, con los cuales se busca comparar las variables seleccionadas y de esta manera evidenciar los posibles cambios y evoluciones socioeconómicos de los pecadores artesanales. En la última parte del trabajo se resaltan las principales conclusiones resultado de los análisis estadísticos, donde identificarán los cambios en la calidad de vida de los pescadores del municipio de Ciénaga.

La literatura existente sobre este tema es muy escasa, dado que muchos trabajos anteriormente realizados plantean problemáticas sobre la pesca, pero muy pocos sobre las condiciones socioeconómicas de los que practican esta actividad. A continuación, se presentarán algunas investigaciones tanto nacionales como internacionales que abordan este tema, y las conclusiones a las cuales llegaron.

En Chile, la pesca artesanal está tomando una forma acelerada en sus procesos, muchas veces conlleva a la pérdida de identidad de algunas comunidades, así lo revela una investigación de Neira (2005). Así mismo, el objetivo se centró en el análisis que enfrentan los habitantes de esta comunidad, frente a los desafíos y problemas en la inserción de la modernización; de igual modo, la investigación también buscaba una caracterización general de la Caleta Queule que, a su vez, representará en datos reales todos los desafíos socioeconómicos propios de la pesca artesanal y que no los lleve a la pérdida de identidad o de su cultura. Cornejo (2002) Realizó un estudio referente a la importancia de la mujer en la actividad pesquera, en el cual recalca que en muchos casos no tiene el rol primario, pero está muy ligada a las actividades subsiguientes, como son principalmente la comercialización.

Sachs (2008), plantea que la pobreza puede ser superada con la realización de cuatro tipos de inversiones básicas, tomando como punto de partida la inversión agrícola, incentivando a los agricultores a la realización de la actividad para conseguir una producción más allá de la necesaria para subsistir. La segunda inversión debe ir reflejada en el sistema de salud y en la disminución de las tasas de mortalidad. Otro punto clave de inversión, es la educación que sirve como herramienta de destrucción de trampas de pobreza y desigualdad brindando oportunidades a personas de bajo de recursos para escalar social 
y económicamente. Por ultimo está la inversión en infraestructura, la cual es vital para el desarrollo del comercio y la industria.

En un estudio a la población de pescadores artesanales de Perú realizado por Galarza y Kámiche (2014), se proponen acciones específicas para que los gobiernos regionales logren impulsar el sector de la pesca artesanal, con actividades tales como inversión en infraestructura, programas de capacitación para la población en mención, incentivos para la formalización y programas de financiamiento, articulándolos con modelos que permitan mejorar la producción, administración y comercialización de sus productos. El objetivo principal fue considerar la pesca artesanal como una oportunidad para el desarrollo regional y no como una actividad de estancamiento.

En el estudio de caso realizado por Pallares (1996), se evidencia diferencia en el poder de negociación que manejan los pescadores dependiendo de diferentes factores. Para el caso de los pescadores que desembarcan en el mercado local, estos han obtenido la destreza para poder realizar transacciones con un comerciante u otro. Pero esto difiere para el caso de los pescadores de los pueblos paralíticos (Bocas de Aracataca, Buena Vista, Nueva Venecia), los cuales dependen del comercializador que por ser propietario de la tienda y casi el $70 \%$ de las canoas que se utilizan para las faenas de pescas, es, en últimas, quien termina imponiendo el precio.

Según Viloria (2008), en su trabajo de extractiva y pobreza en la Ciénaga de Zapatosa, las condiciones sociales de los pescadores son muy desfavorables, en su mayoría debido a que la producción es estacional, y esto ocasiona que los ingresos sean fluctuantes a lo largo del año, y varíen dependiendo de las condiciones climáticas que se originen en la ciénaga. Esto conduce como consecuencia un endeudamiento de los pecadores durante el año, forzándolos a vender su producción al comercializador que les presta dinero, y de esta manera eliminando la posibilidad de ahorrar. En el estudio del INPA realizado en 2002 (citado por Viloria, 2008), se calculó que el ingreso promedio de un pescador es aproximadamente de $\$ 319.000$, similar al salario mínimo de ese año.
Aguilera (2011) afirmó:

La pesca de crustáceos y moluscos que se realiza de manera artesanal por cerca de 5.000 pescadores, de la cual dependen cerca de 20.000 personas que habitan en los siete municipios de la ecorregión CGSM. En Pueblo Nuevo, Pueblo Viejo y Ciénaga hay cerca de 30 asociaciones de primer nivel que agrupan entre 15 y 25 pescadores. Además existen las asociaciones de segundo nivel, entre ellas Gran Pez, Asoisla, Asociénaga, que organizan a las del primer nivel y les gestionan proyectos productivos. A su vez, las de segundo nivel son agrupadas en las de tercer nivel (Asopesca) que canalizan recursos, tales como los de la sobretasa ambiental (Ley 981 de 1994), para desarrollar proyectos productivos entre las comunidades de pescadores y sus familias. (p.23)

En Ciénaga, la pesca artesanal representa una actividad básica, en el cual su forma de captura y el modo de ejercerla han representado una tradición por muchas décadas. Grijalba, Bustos, Posada y Santafé (2012), realizan un acercamiento con las comunidades de pescadores artesanales del departamento del Magdalena y evalúan todo lo relacionado con el desarrollo de la pesca. También proponen un planteamiento no solo desde la óptica investigativa, sino también con la visión de una actividad socioeconómica fundamental para la región; los autores vincularon también a diferentes familias en las etapas desarrolladas, llegando a convertirse en un espacio con sentido social en el que se enfatizó la necesidad de promover la pesca responsable.

\section{Metodología}

Para esta investigación se utilizó la metodología propuesta por Dankhe (1986) en el marco del tipo de investigación descriptiva, que en su condición constituye un punto de partida para el impacto que puedan tener los pescadores artesanales del municipio de Ciénaga, Magdalena. En esta misma línea se establecieron dos variables: $i)$ identificar las características sociales de los pescadores artesanales de este municipio; y ii) determinar a través de una encuesta cocensal cuantos suman sus ingresos económicos. 
Para llegar a los resultados mencionados anteriormente fue necesario hacer un estudio de campo, para lo cual se realizaron 596 encuestas a dicha población de las cuales 397 fueron hechas en el 2011 en el proyecto Caracterización social y económica de los pescadores artesanales de Ciénaga Magdalena. Informe, Santa Marta (Mendoza \& Morón) y el resto en el periodo 2014 (199 encuestas).

Esta encuesta facilita toda la información en tiempo real y que, a su vez, sirve para la interpretación de los resultados mediante el desarrollo del respectivo censo en la población; además de conocer a través de estas estimaciones las características en el nivel de individuos, vivienda y población pescadora del municipio de Ciénaga Magdalena.

\section{- Universo y cobertura}

El censo abarcó la población dedicada a la pesca artesanal para obtener información sobre todas las viviendas, todos los hogares y todas las personas pescadoras. Desde el punto de vista del censo básico y la encuesta cocensal, el nivel de desagregación comprende el sector del Barrio Abajo, 12 de 0ctubre, Kennedy, El Oasis, Puerto Nuevo, Mar de Plata.

\section{- Población objetivo}

Todas las personas, viviendas y hogares cuyos jefes de hogar se dediquen a la pesca artesanal y que residan o lleven a cabo la actividad pesquera en el Mar Caribe y en la Ciénaga Grande de Santa Marta que accede a la cabecera municipal.

\section{- Diseño de instrumentos}

El contenido temático de los cuestionarios, se definieron de acuerdo con los requerimientos institucionales y técnicos referentes a la necesidad de representatividad de la información básica censal. En efecto, fue importante considerar como condición que definió el contenido del formulario básico la información que, por sus características, se constituye en información estratégica para el establecimiento de líneas de base en los procesos de planeación, seguimiento y evaluación de la gestión pública local.
La información obtenida fue tabulada en Excel y luego codificada y graficada a través del paquete estadístico IBM SPSS STATIC, del cual se logró obtener graficas que explican el comportamiento de las variables estudiadas.

\section{Resultados}

El trabajo de campo realizado en los barrios donde residen los pescadores artesanales del municipio (sobre la Ciénaga Grande y el Mar Caribe) permitió obtener información de 199 familias, los cuales se analizarán en este apartado, a la vez que se compararan con los obtenidos en el año 2011.

- Perfil de las familias dedicadas a la pesca artesanal

El género predominante obtenido del trabajo de campo en los barrios donde residen los pescadores artesanales y sus familias correspondientes al municipio de Ciénaga, evidenció la mayoría del género masculino, que representaban el $68,84 \%$ de las personas encuestadas.

Para las encuestas realizadas en el 2014 se observó que la población de pescadores artesanales está conformada plenamente por familias, el número de personas solteras aumentó en aproximadamente $3,11 \%$. El $77,89 \%$ respondió que es casado o vive con su cónyuge en unión libre.

La educación es condición esencial para el desarrollo social, cultural y económico de la región y un derecho consagrado en la Constitución Nacional. En gran medida esta puede explicar el comportamiento de muchas variables interpretadas en el trabajo. Por tal motivo que llega al punto de indagar sobre la educación de los pescadores artesanales y sus familias.

En la Figura 1 se observa el nivel educativo de los encuestados, casi $1 / 4$ de los pescadores artesanales no tienen ninguna clase de estudios educativos. Por otra parte, el 27,66\% dice haber dejado la primaria incompleta, seguido del $18,09 \%$ que representa el bachillerato incompleto, y finalmente solo un $14,89 \%$ de los pescadores son bachilleres. En comparación con los años de estudio 2011-2014 la 
situación ha empeorado, debido a que para el 2011 solo el $20,2 \%$ de la población no tenía ninguna clase de estudios y como se había mencionado anteriormente para el 2014 era del 23,94\% lo que significa que hubo un incremento de $3,74 \%$. Siguiendo la misma idea, para el 2014 no se encontraron técnicos o profesionales dentro del área de la muestra del estudio, lo que indica que estas personas y sus familias cada vez más estas siendo atrapadas en una trampa de pobreza.

Figura 1.

Nivel educativo de los encuestados comparación 2011-2014
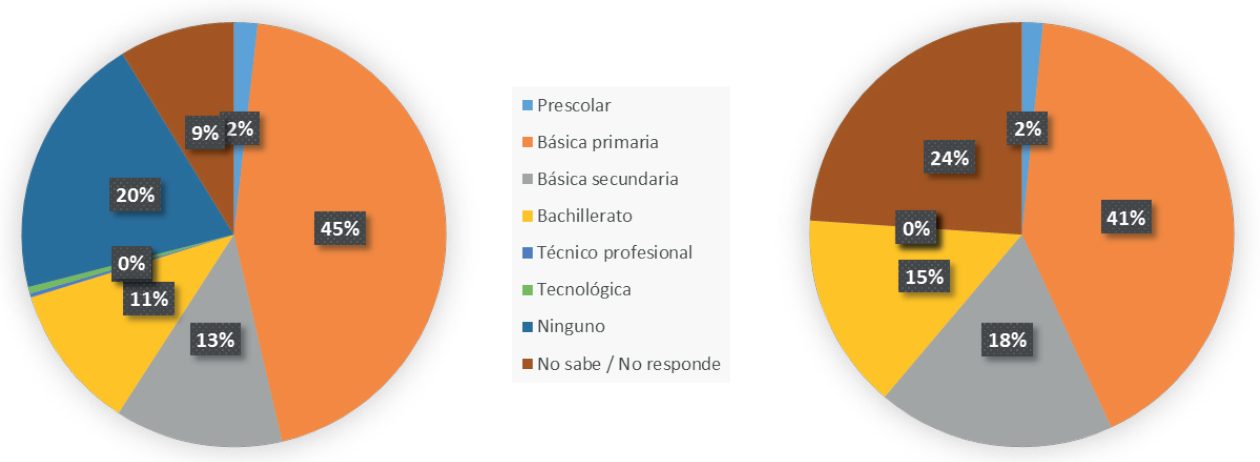

- Prescolar

- Básica primaria

- Básica secundaria

Bachillerato

- Técnico profesional

- Tecnológica

- Ninguno

- No sabe / No responde

Fuente: Morón \& Mendoza (2011, p.18) Fuente: elaboración propia con base en la encuesta aplicada para este estudio

En lo que respecta al nivel educativo de los encuestado, se pudo establecer que para los años 2011 y 2014 el nivel académico con mayor participación era básica primaria con $44,60 \%$ y $41,49 \%$, con una variación negativa del 3,11\% entre el 2011-2014; seguido a esto, para el 2011 el 20,20\% aseguro no haber cursado ningún curso educativo, para el 2014 esta situación empeoró debido a que para ese año el 23,94\% afirmó que no tenía ninguna clase de formación escolar, concluyendo así una desmejora en la educación de los pescadores de la Ciénaga, esto debido a la trampa de pobreza que rodea a este grupo particular de la población.

De acuerdo con las estadísticas obtenidas, se logra establecer que el $17,59 \%$ de la población encuestada no tiene hijas, en tanto que un $26,13 \%$ tiene una hija y de igual forma el $26,13 \%$ tiene dos hijas, un $15,58 \%$ dice tener tres hijas y el $14,57 \%$ confirmó tener entre 4 y ocho hijos. Por otro lado, en la comparación del número de hijas del año 2011-2014 la variación más significativa fue la de no tener ni una hija, dado que en el 2011 el 21\% de la población objeto de estudio dijo no tener hijas, y en la actualidad para la misma respuesta lo dijeron un 17,59\%, lo que significa una variación del 3,41\%, las otras variables permanecieron casi constantes al pasar de los años.

En cuanto al número de hijas que se encuentran estudiando en algún centro educativo para el 2014 es preocupante, dado que para el 2011 el 47\% de las hijas no estaban estudiando y para el 2014 el $50,75 \%$ seguía en la misma situación, lo que significa que a través de los tres años el número de hijas no matriculadas en instituciones educativas ha aumentado un $3,75 \%$, y como segundo dato relevante para el 2011 el 27\% de los encuestados tenían al menos una hija estudiando y en el 2014 el $26,13 \%$, se evidencia claramente que a través del tiempo no ha variado esta situación.

Por otra parte, el $25,13 \%$ de las familias de los pescadores asegura tener dos hijos y el $21,61 \%$ 
tener solo un hijo, así mismo el $21,11 \%$ de los encuestados aseveró no tener hijos. Entre las variaciones y comparaciones del 2011-2014 el único factor significativo fue 3 hijos, dado que este vario más del $7 \%$, puesto que para el 2011 el $10 \%$ de los pescadores artesanales tenía 3 hijos y para el año actual $17,59 \%$ tenía 3 hijos.

Respecto al número de hijos estudiando, cerca de la mitad de los encuestados $(48,74 \%)$ respondió que ninguno de sus hijos se encuentra estudiando, otro $26,63 \%$ aseguró que un hijo suyo está estudiando, mientras que otro $17,69 \%$ dijo que tiene dos hijos adelantando algún estudio. Los resultados arrojados del 2014 no demuestran ningún avance significativo entre el año 2011-2014 en lo que respecta a la educación de los hijos de los pescadores.

- Condiciones de infraestructura y servicios públicos domiciliarios

Específicamente para 2014 el 78,57\% de los encuestados habitan en una vivienda cuya infraestructura se considera como casa, mientras el 10,20\% afirmaron vivir en un apartamento, seguido muy de cerca de los que habitan un tipo de vivienda palafítica. Del 20112014 hubo una variación del $3,47 \%$ de las personas que identificaban su vivienda como casa dado que para el 2011 el 82\% vivían en casas y como se había mencionado anteriormente para la actualidad el 78,57\% identificaron su infraestructura de vivienda como casa.

\section{- Figura 2.}

\section{Tipo de vivienda comparación 2011-2014}
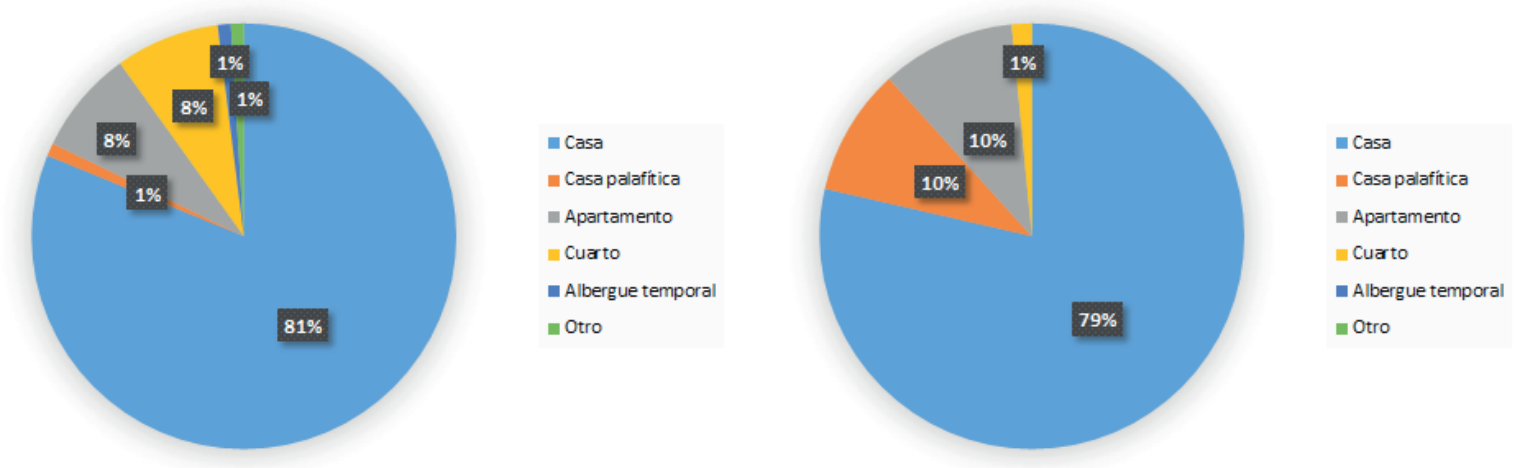

Fuente: Morón E Mendoza (2011, p.23) Fuente: elaboración propia con base en la encuesta aplicada para este estudio

Un aspecto que se puede asociar a las condiciones económicas de los pescadores artesanales del municipio de Ciénaga, es el material predominante de las paredes exteriores de la vivienda, tomando esto en cuenta, no se evidenciaron grandes cambios de los encuestados del 2011 y el 2014, de los cuales observó una variación del 10,08\% de las casas que están construida en bloque, ladrillo, piedra, madera pulida, y un variación del $8 \%$ de los que aseguraron que el material predominante de su casa es la madera burda, tabla, tablón.
- Perfil productivo de las familias dedicadas a la pesca artesanal

La situación económica de las familias de los pescadores artesanales se midió por medio de sus ingresos mensuales, esta ha tenido una variación positiva del 2011 hasta el 2014, dado que el porcentaje de familias que tienen ingresos mensuales entre $\$ 50.000$ y $\$ 100.000$, y entre $\$ 120.000$ a $\$ 300.000$ pesos a disminuido un $13,48 \%$, porcentaje que se ve reflejado en las familias que ganan entre $\$ 310.000$ 
y $\$ 560.000$, y entre $\$ 600.000$ y $\$ 1.200 .000$. Por último, las familias que ganan entre $\$ 1.300 .000$ y $\$ 2.000 .000$ no tuvieron un cambio significativo entre los años estudiados, esto debido a que la multinacional carbonera DRUMMOND no ha seguido vinculando a los pescadores.

\section{-1Figura 3.}

\section{Ingresos familiares mensuales comparación 2011-2014}
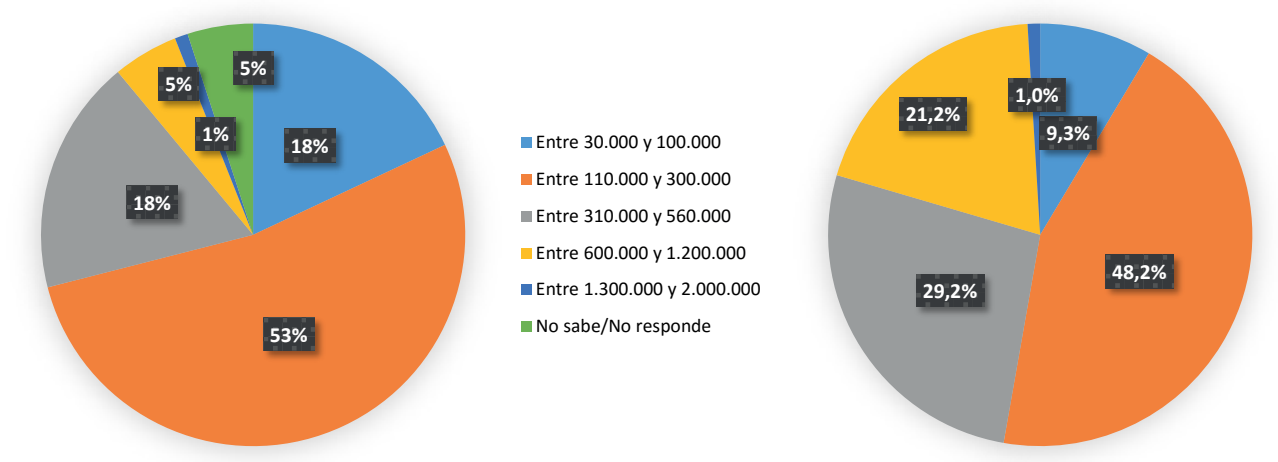

- Entre 30.000 y 100.000 - Entre 110.000 y 300.000 - Entre 310.000 y 560.000 $\square$ Entre 600.000 y 1.200 .000 - Entre 1.300 .000 y 2.000 .000 No sabe/No responde

Fuente: Morón E Mendoza (2011, p.30) Fuente: elaboración propia con base en la encuesta aplicada para este estudio

De igual manera, se consultó sobre el número de personas que trabajan en la familia, para el 2014 del $100 \%$ de los encuestados el $48,45 \%$ confirma que por lo menos un miembro de la familia aporta ingresos por medio del trabajo, seguido de un $30,08 \%$ que equivale a dos personas que trabajan y por último el $15,46 \%$ indica que tres o más miembros trabajan en su núcleo familiar. En comparación con el 2011, se puede observar que hubo una fuerte variación en la respuesta, donde solo dos personas trabajan en la familia, debido a que en el 2011 corresponde un $23 \%$ y en el año actual $36,08 \%$, esto puede estar explicado porque en el 2011 había opción de responder No Sabe/No Responde, la cual tuvo un 14\%, mientras que en la encuesta actual esa opción de respuesta no estaba.

En lo que respecta a la proporción del número de personas que tienen a cargo, para el 2014 el 56,85\% de los encuestados respondieron que tenían tres o más personas a cargo, mientras que el $42,64 \%$ respondió que está a cargo de una o dos personas, el restante no está a cargo de ninguna persona. Estudiando la variación del 2011-2014 de la variable anteriormente mencionada se puede observar una disminución del $7,15 \%$ la cual se ve explicada en gran parte por una 0 dos personas que tiene a cargo, dado que uno y dos personas a cargo del 2011 suman el $28 \%$ mientras que en el 2014 suman 42,64\%.

La investigación evidencia que solo una parte de la población es propietaria de la embarcación donde se realiza las labores de pesca $(25,40 \%)$, mientras que un $35,57 \%$ afirmó que era arrendada. Se demostró un claro aumento en el número de propietarios de embarcaciones utilizadas para faena.

Por otro lado, quienes realizan la comercialización de la producción pescada, respondieron en su mayoría que lo hacen en el mercado público $(55,85 \%)$. La comercialización con familiares corresponde al $15,43 \%$ y con vecinos un $4,47 \%$. Todas las variables demostraron un claro aumento, con respecto al 2011, pero esto se debido a que la proporción de los encuestados que escogieron la opción No sabe/ No responde como repuesta en el 2011 es del 22\%, a diferencia de del 2014 en la cual fue de cero. 


\section{JFigura 4.}

\section{Propiedad de la embarcación donde realiza las labores de pesca}
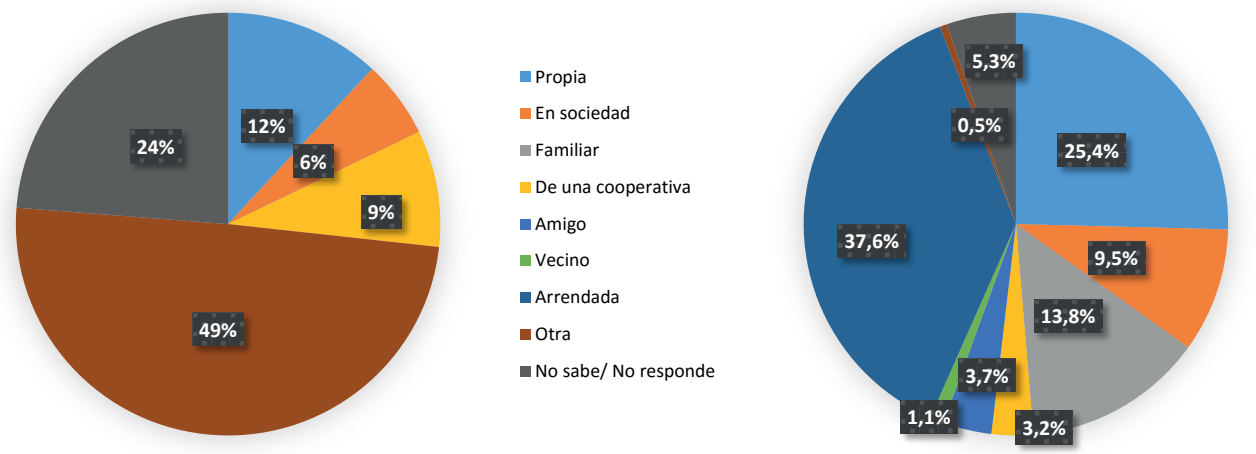

- Propia

En sociedad

- Familiar

De una cooperativa

Amigo

Vecino

- Arrendada

Otra

- No sabe/ No responde

Fuente: Morón E Mendoza (2011, p.33) Fuente: elaboración propia con base en la encuesta aplicada para este estudio

\section{- Figura 5.}

\section{Comercialización de la producción pescada comparación 2011-2014}
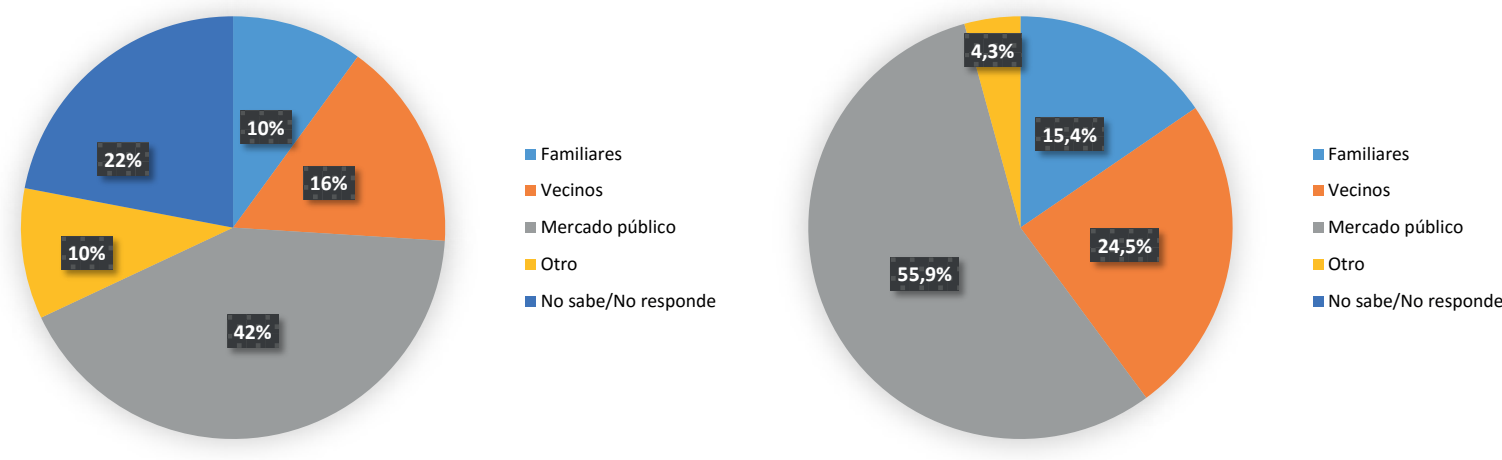

Fuente: Morón \& Mendoza (2011, p.35) Fuente: elaboración propia con base en la encuesta aplicada para este estudio

- Afiliación a Organizaciones de la Sociedad Civil (OSC)

Con el propósito de identificar si los pescadores artesanales se encuentran afiliados a algún tipo de organizaciones sociales 0 civiles, se le pregunta a la población de caso de estudio si está afiliada a una OSC, a lo que el $50,81 \%$ responde que no, el $23,78 \%$ dice que sí y el restante 25,41 reconocen no saber. En comparación con los años de estudio se logra identificar grandes variaciones en no pertenecer a alguna OSC, expuesto por una diferencia del 2011-2014 de 18,19\%, logrando que para el 2011 el $69 \%$ de los encuestados estaba afiliado a alguna
OSC. Además, otra gran variación se identifica en la respuesta de No sabe/No responde del $24,41 \%$ de diferencia entre los años de estudio, ya que para el 2011 solo un 1\% de la población se identificó con esta respuesta, a diferencia de la actual, ya antes mencionada.

\section{Discusión}

En los resultados obtenidos se observaron similitudes con trabajos previos, como en el estudio realizado por Cotto y Marttín (2008), en el cual se evidencia que los retornos que reciben los pescadores artesanales por su trabajo no son suficientes 
para suplir las necesidades básicas de su núcleo familiar. Esta situación se observa de una manera más notoria en las comunidades de la costa caribe por la carente infraestructura que facilite la comunicación y un deterioro de la cobertura de programas institucionales.

Dicho escenario se observa en las familias de la comunidad pesquera del municipio de Ciénaga, las cuales no demostraron grandes avances en su calidad de vida; por el contrario, en algunos casos, como lo fue el número de hijas que se encuentran estudiando en un instituto educativo, nos encontramos con que este es porcentualmente menor en el 2014 con respecto al 2011.

Para los pescadores artesanales de cualquier comunidad es de gran importancia o ayuda ser el propietario de embarcación, como se demostró en el estudio de caso de Pallares (1996), debido a que esto le permite tener más poder de negociación a la hora de imponer el precio. Desde este punto de vista, los pecadores de Ciénaga tuvieron un claro desarrollo al incrementar el número de personas propietarias de su propia embarcación el cual llego a duplicarse, pero cabe resaltar que, aunque hubo un aumento relativo en el año 2014 con respecto al 2011, todavía sigue siendo muy poco en términos generales, por lo cual se debe proporcionar mayores ayudas para que obtengan mejores condiciones de trabajo.

En general, la mayoría de los pescadores artesanales del municipio de Ciénaga trabajan en gran parte a nivel individual y esto no contribuye a mejorar su calidad de vida, por consiguiente, es necesario del apoyo de campañas de formalización de agremiaciones para desarrollar la actividad pesquera más organizadas y tendientes a la comercialización para su mismo beneficio.

Además, Agüero (1992) en su trabajo logra distinguir unos rasgos característicos en la pesca artesanal de los países de América Latina, entre los que destacamos: la fuerte dependencia que tienen de las cadenas de comercialización, la desarticulación social y económica con el resto del país y la incipiente organización gremial, rasgos que como se observan siguen presentándose hasta la fecha, mostrando en términos generales la poca evolución que ha tenido esta actividad económica y quienes se dedican a ella.

\section{Conclusión}

El trabajo de investigación evidenció desmejoras en la calidad de vida de los pescadores y su núcleo familiar, mostrando retrocesos en los niveles académicos alcanzados, y aumentos de perturbaciones sociales producidas en los métodos de eliminación de basura la cual se incrementaron en el 2014 con respecto al 2011.

Así mismo, se refleja estancamiento o poco progreso en la calidad de vida de la mayoría los pescadores y sus familias, situación alarmante a la que se le debe prestar la atención necesaria para un pronto mejoramiento de la situación socioeconómica del grupo de estudio.

En los resultados de la investigación se esperaba encontrar y comprobar, que la situación de vida y características de los pescadores artesanales y sus familias mejoraran, puesto que a medida que pasan los años, la evolución en el bienestar de la población tiende a mejorar. Es de vital importancia llevar un constante seguimiento de evolución en los pescadores y sus familias, para poder obtener mayor información de los obstáculos para salir de la trampa de pobreza que azota a esta población.

Dadas las condiciones que se han estudiado en este periodo de tiempo, se puede afirmar que sin una intervención del Estado o del sector privado, las condiciones de vida en un futuro para esta población seguirán siendo desfavorables. Es claro que se necesita gran inversión en variables como educación, puesto que esta es una de las claves del desarrollo en la sociedad actual.

Se requiere de manera urgente que las nuevas generaciones de los pescadores artesanales de Ciénaga, Magdalena y todo el núcleo familiar, pueda tener un acceso a la educación de carácter definitivo, ya que este es, el motor de desarrollo para las regiones y donde también haya una absoluta cobertura diseñada por la administración pública que garantice los niveles de escolaridad de sus habitantes. 
Es de valía resaltar la importancia de aumentar el número de encuestados o muestra de la población total en futuros trabajos para generar mayor confiabilidad de resultados a obtener, logrando así obtener mayores probabilidades de acierto en la evolución de las variables estudiadas y disminuir el sesgo de información.

\section{Referencias Bibliográficas}

Agüero, M. (1992) La Pesca Artesanal en América Latina: Una Visión Panorámica.

Aguilera, M. (2011). Habitantes del agua: El complejo lagunar de la Ciénaga Grande de Santa Marta. Banco de la Republica. Recuperado de http://www.banrep.gov.co/sites/ default/files/publicaciones/archivos/DTSER-144.pdf

Cornejo, A. (2002). La mujer en la pesca artesanal argentina: estado actual y perspectivas.

Cotto, A., \& Marttín, F. (2008). Estrategias para el Desarrollo sostenible de la Pesca Artesanal, La seguridad Alimentaria y Reducción de la Pobreza de la Familias Vinculadas. INPESCA.

DANE. (2005). Censo General 2005. Recuperado de http://www. dane.gov.co/index.php/esp/poblacion-y-registros-vitales/ censos/censo-2005

DANE. (2005). Necesidades Básicas Insatisfechas -NBI. Recuperado de http://www.dane.gov.co/index.php/ estadisticas-sociales/necesidades-basicas-insatisfechas-nbi
Dankhe. (1986). Diferentes diseños. Tipos de investigación.

Galarza, E., \& Kámiche, J. (2014) Pesca artesanal: una oportunidad para el desarrollo. Universidad del Pacífico. Recuperado de http://agenda2014.pe/publicaciones/ agenda2014-pesca-artesanal.pdf

Grijalba, M., Bustos, D., Posada, C., \& Santafé, A. (2012). La pesca artesanal marítima del departamento del Magdalena: Una visión desde cuatro componentes. (Primera ed.). Santa Marta, Colombia: Fundación Universidad de Bogotá Jorge Tadeo Lozano.

Mendoza, J., \& Morón, J. (2011). Caracterización Social y Económica de los Pescadores Artesanales de Ciénaga Magdalena. Informe, Santa Marta.

Pallares, 0. (1996). Análisis de la cultura de la pobreza en una comunidad de pescadores. Estudio de caso, pueblo viejo (Magdalena). Universidad del Norte. Recuperado de http://ciruelo.uninorte.edu.co/pdf/invest_desarrollo/4/ analisis_de_la_cultura_de_la_pobreza_en_una_comunidad_de_pescadores.pdf

Sachs, J. (2008). Economía Para un Pueblo Abarrotado (Vol. I). (R. Pérez, Trad.) Barcelona, España: SIAGSA.

Universidad de la Republica. (2011). Estudio de la Cadena de Comercialización de la Pesca Artesanal del Rio Uruguay y Alternativas Productivas de Diversificación.

Viloria, J. (2008). Economía extractiva y pobreza en la ciénaga de Zapatosa. Banco de la Republica. Recuperado de http://www.banrep.gov.co/docum/Lectura_finanzas/pdf/ DTSER-103.pdf. 